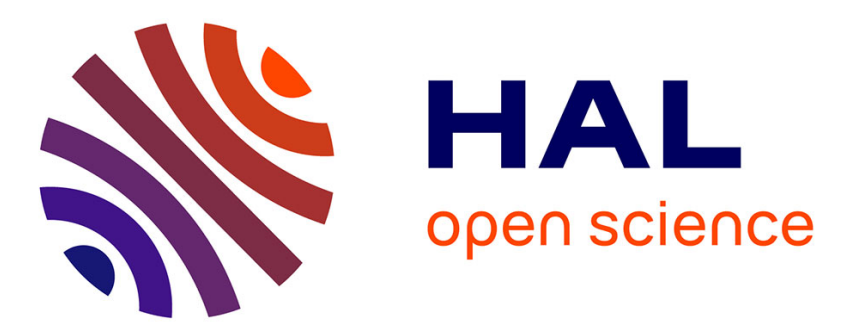

\title{
From the Boltzmann description for mixtures to the Maxwell-Stefan diffusion equations
}

\author{
Francesco Salvarani
}

\section{To cite this version:}

Francesco Salvarani. From the Boltzmann description for mixtures to the Maxwell-Stefan diffusion equations. Particle Systems and PDE's VIII, Dec 2019, Lisbonne, Portugal. hal-02928227v2

\section{HAL Id: hal-02928227 \\ https://hal.science/hal-02928227v2}

Submitted on 13 Oct 2020

HAL is a multi-disciplinary open access archive for the deposit and dissemination of scientific research documents, whether they are published or not. The documents may come from teaching and research institutions in France or abroad, or from public or private research centers.
L'archive ouverte pluridisciplinaire HAL, est destinée au dépôt et à la diffusion de documents scientifiques de niveau recherche, publiés ou non, émanant des établissements d'enseignement et de recherche français ou étrangers, des laboratoires publics ou privés. 


\title{
From the Boltzmann description for mixtures to the Maxwell-Stefan diffusion equations
}

\author{
Francesco Salvarani
}

\begin{abstract}
This article reviews some recent results on the diffusion limit of the Boltzmann system for gaseous mixtures to the Maxwell-Stefan diffusion equations.
\end{abstract}

Key words: Boltzmann system; Maxwell-Stefan equations; cross-diffusion.

\section{Introduction}

The Maxwell-Stefan system [25, 29] has been derived in the 19th century for describing the diffusion behaviour of multicomponent gaseous mixtures. These equations are very popular in the context of chemical engineering [30] and have been extensively studied in the 20th century from the numerical point of view [17, 18]. Starting from [10] and [12], a renewed interest in the Maxwell-Stefan equations arose in the mathematical community and several colleagues have worked for better understanding the mathematical properties of these equations.

The goal of this article is to review the recent literature devoted to a specific topic: the Maxwell-Stefan limit of kinetic equations for gaseous mixtures.

The starting point, in the case of non-reacting mixtures, is the standard extension of the Boltzmann equation [5], derived by Ludwig Boltzmann in 1872. However, many other variants of this model exist: we quote, for example, [28] and [26], which provide two very popular examples of kinetic equations for gaseous mixtures. In the reactive case, the situation is similar. Several models have been proposed (for example, [21]), but the Maxwell-Stefan asymptotics has been mainly studied starting from the models proposed and studied in [16] and in [24].

Francesco Salvarani

Léonard de Vinci Pôle Universitaire, Research Center, F-92916 Paris La Défense, France \& Università degli Studi di Pavia, Dipartimento di Matematica, I-27100 Pavia, Italy, e-mail: francesco.salvarani@unipv.it 
The structure of the article is the following. We first introduce the MaxwellStefan equations and two of the main kinetic models for non-reactive and reactive mixtures. Then, we consider the diffusive limit of the non-reactive system to the Maxwell-Stefan equations at the formal level. The scaling has been introduced in [13], and subsequently developed in [11] and [23]. After describing these formal results, we summarize the result by Bondesan and Briant [8], which provides a rigorous description of the limiting procedure. The next chapter is devoted to the reactive case, and is essentially based on the recent articles [2], [3] and [4]. We conclude this review by quoting the most recent literature on some strictly related subjects.

\section{The Maxwell-Stefan equations}

The classical Maxwell-Stefan system describes the diffusive behaviour of an ideal gaseous mixture, composed by $\mathscr{I} \in \mathbb{N}^{*}$ species. Let $\Omega \subset \mathbb{R}^{3}$ be a bounded domain with regular boundary. The unknowns of the problem are the $\mathscr{I}$ densities $c_{i}: \mathbb{R}^{+} \times$ $\Omega \rightarrow \mathbb{R}$ and the $\mathscr{I}$ fluxes $J_{i}: \mathbb{R}^{+} \times \Omega \rightarrow \mathbb{R}^{3}$, which obey to the following crossdiffusion relationships:

$$
\left\{\begin{array}{l}
\partial_{t} c_{i}+\nabla_{x} \cdot J_{i}=0, \\
\nabla_{x} c_{i}=-\sum_{j \neq i} k_{i j}\left(c_{j} J_{i}-c_{i} J_{j}\right) .
\end{array} \quad(t, x) \in \mathbb{R}^{+} \times \Omega\right.
$$

Here we use a notation which is more adapted from the mathematical point of view with respect to the traditional notation used in the applications: $k_{i j}=1 / \mathrm{Ð}_{i j}$, where the constants $\bigoplus_{i j}$ are suitable symmetric binary diffusion coefficients (i.e. $\bigoplus_{i j}=\bigoplus_{j i}$ for all $i, j=1, \ldots, \mathscr{I})$.

Because of the symmetry of the whole set of binary diffusion coefficients, it is immediate to see that the flux-gradient relationships in (1) are linearly dependent. For this reason, an additional constitutive equation has to be added to (1) in order to have a closed set of equations. The usual assumption - which guarantees the diffusive behaviour of the mixture - is the equimolar diffusion condition

$$
\sum_{i=1}^{\mathscr{g}} J_{i}=0
$$

Usually, the system of PDEs (1) is supplemented with appropriate initial and boundary conditions. When dealing with isolated systems, it is standard to suppose that the solution of (1)-(2) satisfies homogeneous Neumann boundary conditions:

$$
c_{i}(0, x)=c_{i}^{\mathrm{in}}(x) \in L^{\infty}(\Omega),\left.\quad J_{i}(t, x) \cdot n_{x}\right|_{(t, x) \in(0, \infty) \times \partial \Omega}=0
$$

for all $i=1, \ldots, \mathscr{I}$, where $n_{x} \in \mathbb{S}^{2}$ is the outward normal unit vector to the domain $\Omega$ starting from a given point $x \in \partial \Omega$. The Maxwell-Stefan system (1)-(2) is often 
written in terms of molar fraction. In this case, it is supposed that $c_{i}^{\text {in }} \geq 0$ and that

$$
\sum_{i=1}^{\mathscr{I}} c_{i}^{\mathrm{in}}(x)=1
$$

This normalization property is conserved by the time evolution of the system. It means that

$$
\sum_{i=1}^{\mathscr{I}} c_{i}(t, x)=1 \text { for a.e. }(t, x) \in \mathbb{R}^{+} \times \Omega
$$

\section{The Boltzmann system for monatomic inert gaseous mixtures}

The Boltzmann system for a mixture of ideal monatomic non-reacting gases $\mathscr{A}_{i}$, $i=1, \ldots, \mathscr{I}$ with $\mathscr{I} \geq 2$, subject to elastic mechanical collisions, describes the time evolution of the species by means of $\mathscr{I}$ distribution functions $f_{i}$, defined on the phase space of the system. The particles of each species are identical and can be modelled as point masses. Their main physical quantities are therefore the mass $m_{i} \in \mathbb{R}^{+}$and the velocity $v \in \mathbb{R}^{3}$. The standard assumption on the density function is of $L^{1}$-type, i.e. $f_{i}(t, \cdot, \cdot) \in L_{\text {loc }}^{1}\left(\mathbb{R}_{x}^{3} ; L^{1}\left(\mathbb{R}^{3}\right)\right)$, for all $t \in \mathbb{R}^{+}$. This requirement guarantees that the system has finite mass. In what follows, we assume that the system is isolated, so that no external force acts on the particles. Moreover, we suppose that only elastic binary collisions are allowed. Therefore, momentum and kinetic energy are conserved during the interaction process. Under the previous hypotheses, the time evolution of the distribution functions $f_{i}$ is governed by the Cauchy problem

$$
\begin{aligned}
\partial_{t} f_{i}+v \cdot \nabla_{x} f_{i} & =\sum_{j=1}^{\mathscr{I}} Q_{i j}\left(f_{i}, f_{j}\right) & \text { for }(t, x, v) & \in(0, \infty) \times \mathbb{R}^{3} \times \mathbb{R}^{3} \\
f_{i}(0, x, v) & =f_{i}^{\text {in }}(x, v) & \text { for }(x, v) & \in \mathbb{R}^{3} \times \mathbb{R}^{3}
\end{aligned}
$$

for each $i=1, \ldots, \mathscr{I}$, where $Q_{i j}(\cdot, \cdot)$ denotes the bilinear integral operator describing the collisions of molecules of species $\mathscr{A}_{i}$ with molecules of species $\mathscr{A}_{j}$.

A given particle follows a rectilinear trajectory, until it collides with another particle. When two particles belonging to the species $\mathscr{A}_{i}$ and $\mathscr{A}_{j}$, with masses $m_{i}, m_{j}$, and pre-collisional velocities $v^{\prime}, v_{*}^{\prime}$ collide, they exchange kinetic energy and momentum. A microscopic collision is a local in space and instantaneous in time phenomenon. It modifies the velocities of the particles, which become $v$ and $v_{*}$, under the constraint of conserving the total kinetic energy and the total momentum:

$$
m_{i} v^{\prime}+m_{j} v_{*}^{\prime}=m_{i} v+m_{j} v_{*}, \quad \frac{1}{2} m_{i}\left|v^{\prime}\right|^{2}+\frac{1}{2} m_{j}\left|v_{*}^{\prime}\right|^{2}=\frac{1}{2} m_{i}|v|^{2}+\frac{1}{2} m_{j}\left|v_{*}\right|^{2} .
$$

The previous equations can be easily inverted: 
$v^{\prime}=\frac{1}{m_{i}+m_{j}}\left(m_{i} v+m_{j} v_{*}+m_{j}\left|v-v_{*}\right| \sigma\right), v_{*}^{\prime}=\frac{1}{m_{i}+m_{j}}\left(m_{i} v+m_{j} v_{*}-m_{i}\left|v-v_{*}\right| \sigma\right)$,

where $\sigma \in \mathbb{S}^{2}$ is a unit vector which allows to keep into account the two degrees of freedom arising in inverting (7).

If $f_{i}$ and $f_{j}$ are two nonnegative functions, the operator describing the collisions between molecules of species $\mathscr{A}_{i}$ and molecules of species $\mathscr{A}_{j}$ is defined by

$$
Q_{i j}\left(f_{i}, f_{j}\right)(v):=\iint_{\mathbb{R}^{3} \times \mathbb{S}^{2}} B_{i j}\left(v, v_{*}, \sigma\right)\left[f_{i}\left(v^{\prime}\right) f_{j}\left(v_{*}^{\prime}\right)-f_{i}(v) f_{j}\left(v_{*}\right)\right] \mathrm{d} \sigma \mathrm{d} v_{*} .
$$

The expressions of $v^{\prime}$ and $v_{*}^{\prime}$, are given by (8).

The quantities $B_{i j}(i, j=1, \ldots, \mathscr{I})$ are usually called cross sections. They satisfy the Galilean invariance and the microreversibility assumptions

$$
B_{i j}\left(v, v_{*}, \sigma\right)=B_{j i}\left(v_{*}, v, \sigma\right)=B_{i j}\left(v^{\prime}, v_{*}^{\prime}, \sigma\right) .
$$

Because of the Galilean invariance, it is possible to prove that the collision kernels $B_{i j}$ only depend on the modulus of the relative velocity and on the cosine of the deviation angle:

$$
B_{i j}\left(v, v_{*}, \sigma\right)=B_{i j}\left(\left|v-v_{*}\right|, \cos \theta\right) \quad \text { with } \quad \cos \theta=\frac{v-v_{*}}{\left|v-v_{*}\right|} \cdot \sigma .
$$

The choice of the collision kernels $B_{i j}$ has a deep influence on the properties of the Boltzmann equation. In the classical elastic case,

$$
B_{i j}\left(\sigma, v-v_{*}\right)=K_{i j}\left|v-v_{*}\right|, \quad K_{i j}>0,
$$

for a gas of three-dimensional hard spheres. This type of cross section is called hardsphere cross section. When the binary forces between particles are proportional to the inverse of the $s$-th power of the relative distance (for example, $s=2$ corresponds to the gravitational force or to the Coulomb force and $s=7$ corresponds to the Van der Waals interaction, see [31] for more details), $B_{i j}$ can be factorized:

$$
B_{i j}\left(\sigma, v-v_{*}\right)=\Phi_{i j}\left(\left|v-v_{*}\right|\right) b_{i j}(\cos \theta) .
$$

In particular, in three space dimensions,

$$
\Phi_{i j}\left(\left|v-v_{*}\right|\right)=\left|v-v_{*}\right|^{\gamma}, \quad \gamma=(s-5) /(s-1),
$$

and all the $b_{i j}$ are locally smooth functions with a non integrable singularity when $\theta \rightarrow 0$ :

$$
b_{i j}(\cos \theta) \sin \theta \sim K_{i j} \theta^{-(1+\eta)}, \quad \eta=2 /(s-1) .
$$

The quantities $\Phi_{i j}$ are known in the literature as the kinetic collision kernels, and the terms $b_{i j}$ are the angular collision kernels. The family of collision kernels 
whose kinetic term has the form $\Phi_{i j}\left(\left|v-v_{*}\right|\right)=\left|v-v_{*}\right| \gamma$ is usually classified in the literature in three sub-classes: hard potentials, when $\gamma>0$, soft potentials, when $\gamma<0$, and Maxwellian potentials when $\gamma=0$.

For simplifying the mathematical treatment of the angular cross section, Grad introduced an angular cut-off assumption, by supposing that angular collision kernel is integrable with respect to the angular variable $[19,20]$.

The link between the macroscopic description and the kinetic formulation is given by the moments of the distribution functions $f_{i}(t, x, v)$. In particular, the number densities $c_{i}$, mass densities $\rho_{i}$, mean velocities $u_{i}$ and kinetic temperatures $T_{i}$ of each species are respectively given by

$$
\begin{gathered}
c_{i}(t, x)=\int_{\mathbb{R}^{3}} f_{i}(t, x, v) \mathrm{d} v, \\
\rho_{i}(t, x)=m_{i} n_{i}(t, x), \\
u_{i}(t, x)=\frac{1}{c_{i}(t, x)} \int_{\mathbb{R}^{3}} v f_{i}(t, x, v) \mathrm{d} v, \\
T_{i}(t, x)=\frac{1}{3 k_{B} c_{i}(t, x)} \int_{\mathbb{R}^{3}} m_{i}\left|v-u_{i}(t, x)\right|^{2} f_{i}(t, x, v) \mathrm{d} v,
\end{gathered}
$$

where $k_{B}=1.380649 \times 10^{-23} \mathrm{~m}^{2} \mathrm{~kg} \mathrm{~s}^{-2} \mathrm{~K}^{-1}$ is the Boltzmann constant.

It is important to underline that the Boltzmann constant has an exact value. It is indeed one of the seven defining constants in the 2019 redefinition of SI base units. In particular, the value of the Boltzmann constant $k_{B}$ allows to define the unit of temperature $T$ (the kelvin), in terms of the SI unit of energy (the joule), because it is the proportionality constant between the thermodynamic temperature and the average thermal energy of a gas. Unfortunately, the numerical value of the Boltzmann constant has not been, in the mathematical community, the focus of attention and often, for simplifying the computations, many articles and books simply suppose that $k_{B}=1$. In the author's opinion, however, this attitude should change because of the central meaning of the Boltzmann constant as defining constant of the SI base units, and therefore $k_{B}$ should explicitly appear in all the computations. This approach would allow moreover an easier transfer of the mathematical studies in kinetic theory to other scientific communities.

A central role in the study of kinetic equations and systems is played by local and global Maxwellians (i.e. Gaussians in the language of kinetic theory).

In three space dimensions, Local Maxwellians have the form

$$
M\left(c_{i}^{*}, u_{i}^{*}, T_{i}^{*}\right)=c_{i}^{*}(t, x)\left(\frac{m_{i}}{2 \pi k_{B} T_{i}^{*}(t, x)}\right)^{3 / 2} e^{-m_{i} \frac{\left|v-u_{i}^{*}(t, x)\right|^{2}}{2 k_{B} T_{i}^{*}(t, x)}}
$$

for some space-time dependent functions $c_{i}^{*}, u_{i}^{*}$ and $T_{i}^{*}(1 \leq i \leq \mathscr{I})$. Global Maxwellians have the same structure as local Maxwellians, but the quantities $c_{i}^{*}$, $u_{i}^{*}$ and $T_{i}^{*}$ are constant in space and time. It is clear that 


$$
\begin{gathered}
c_{i}(t, x)=\int_{\mathbb{R}^{3}} M\left(c_{i}, u_{i}, T_{i}\right) \mathrm{d} v \\
u_{i}(t, x)=\frac{1}{c_{i}(t, x)} \int_{\mathbb{R}^{3}} v M\left(c_{i}, u_{i}, T_{i}\right) \mathrm{d} v \\
T_{i}(t, x)=\frac{1}{3 k_{B} c_{i}(t, x)} \int_{\mathbb{R}^{3}} m_{i}\left|v-u_{i}(t, x)\right|^{2} M\left(c_{i}, u_{i}, T_{i}\right) \mathrm{d} v
\end{gathered}
$$

in agreement with Equations (11)-(14).

It is possible to prove that, in the mono-species case, a local Maxwellian is a local equilibrium of the system for any choice of velocity and temperature. However, when the mixture is composed of more than one species, the local Maxwellian state is still an equilibrium for the whole system only when the velocities and the kinetic temperatures of the species composing the mixture are exactly the same.

\section{The Boltzmann system for polyatomic reactive or non-reactive gas mixtures}

The model described in the previous section is designed for mixtures of monatomic gases. However, when the species which compose the mixture are of polyatomic type, it is not possible to neglect the additional - rotational and vibrational - degrees of freedom induced by the polyatomic structure of the molecules. In this case, not only the kinetic energy, but also the internal energy of the molecules must be taken into account in the energy balance. Moreover, when chemical reactions are allowed, the collisions may modify the species involved in the process: the species of the incoming particles may be different from the species of the outgoing particles. This behaviour needs to be carefully modelled both at the level of the binary encounters and at the level of the time evolution of the density functions.

In this Section, we describe the kinetic system proposed in [16], based on the Borgnakke-Larsen procedure [9], which describes a mixture of polyatomic gases, either of reactive or non-reactive type. The model is defined on an enlarged phasespace: the independent variables of the unknowns $f_{i}$ have the physical meanings of time $\left(t \in \mathbb{R}^{+}\right)$, space $\left(x \in \mathbb{R}^{3}\right)$, velocity $\left(v \in \mathbb{R}^{3}\right)$ and internal energy $\left(I \in \mathbb{R}^{+}\right)$.

Note that the internal energy is defined on $\mathbb{R}^{+}$. This choice allows to "forget" the complicate structure of the discrete energy levels predicted by quantum mechanics, once implemented a strategy for capturing the correct macroscopic behaviour of the mixture. This goal is achieved by introducing a set of suitable measures $\varphi_{i}(I) \mathrm{d} I$, which allow to relate, in a simple and computable way, the Boltzmann level with the macroscopic parameters of the corresponding reactive fluid equations and guarantee that the model is consistent at the macroscopic level, with the energy law of virtually any type of polyatomic gas.

Among the various possible dynamics of a chemically reacting mixture, the authors of [16] have chosen to describe a mixture of four species $A_{1}, A_{2}, A_{3}$ and $A_{4}$, 
which may react in a reversible way through the formula

$$
A_{1}+A_{2} \leftrightharpoons A_{3}+A_{4}
$$

For each species $A_{i}$, with $i=1,2,3,4$, the authors introduce the corresponding distribution function $f_{i}=f_{i}(t, x, v, I)$, the molecular mass $m_{i}$, the chemical binding energy $E_{i}$ and the weight $\varphi_{i}(I)$. During a chemical reaction of type (16), the conservation of mass requires that

$$
m_{1}+m_{2}=m_{3}+m_{4}=M,
$$

and the balance of binding energies, i.e.

$$
E=E_{3}+E_{4}-E_{1}-E_{2},
$$

means that the forward reaction $A_{1}+A_{2} \rightarrow A_{3}+A_{4}$ is endothermic when $E>0$ or exothermic if $E<0$.

In this description, the moments of the distribution function $f_{i}(t, x, v, I)$ are defined in $L^{1}\left(\varphi_{i}(I) \mathrm{d} I \mathrm{~d} v\right)$. In particular, the number density $c_{i}$, mass density $\rho_{i}$, mean velocity $u_{i}$ and temperature $T_{i}$ of each species are respectively given by

$$
\begin{gathered}
c_{i}(t, x)=\int_{\mathbb{R}^{3}} \int_{0}^{+\infty} f_{i}(t, x, v, I) \varphi_{i}(I) \mathrm{d} I \mathrm{~d} v, \quad \rho_{i}(t, x)=m_{i} c_{i}(t, x), \\
u_{i}(t, x)=\frac{1}{c_{i}(t, x)} \int_{\mathbb{R}^{3}} \int_{0}^{+\infty} v f_{i}(t, x, v, I) \varphi_{i}(I) \mathrm{d} I \mathrm{~d} v, \\
T_{i}(t, x)=\frac{1}{3 k_{B} c_{i}(t, x)} \int_{\mathbb{R}^{3}} \int_{0}^{+\infty} m_{i}\left|v-u_{i}(t, x)\right|^{2} f_{i}(t, x, v, I) \varphi_{i}(I) \mathrm{d} I \mathrm{~d} v .
\end{gathered}
$$

The possible collisions - of binary type - between the molecules of the mixtures are either elastic or reactive.

In the case of elastic encounters, the particles involved in the collision modify their velocities and internal energies, but the chemical composition of the particles is unchanged. If we denote the velocities and internal energies of the colliding particles before the collision by $v_{i}, v_{j}$ and $I_{i}, I_{j}$ respectively, and their corresponding postcollisional values by $v_{i}^{\prime}, v_{j}^{\prime}$ and $I_{i}^{\prime}, I_{j}^{\prime}$, the conservation laws of momentum and total energy for elastic collisions are given by

$$
\begin{aligned}
m_{i} v_{i}+m_{j} v_{j} & =m_{i} v_{i}^{\prime}+m_{j} v_{j}^{\prime} \\
\frac{1}{2} m_{i}\left|v_{i}\right|^{2}+I_{i}+\frac{1}{2} m_{j}\left|v_{j}\right|^{2}+I_{j} & =\frac{1}{2} m_{i}\left|v_{i}^{\prime}\right|^{2}+I_{i}^{\prime}+\frac{1}{2} m_{j}\left|v_{j}^{\prime}\right|^{2}+I_{j}^{\prime} .
\end{aligned}
$$

In particular, $i \neq j$ for bi-species collisions and $i=j$ for mono-species collisions.

Reactive collisions occur among particles of species $A_{1}, A_{2}$ or $A_{3}, A_{4}$. In this case, the velocities and the internal energies are modified by the collision and, moreover, the reactants are transformed into the products of the reaction. By following the notation of [2], the rearrangement of mass and the redistribution of chemical binding energy are formalized as follows. 
If $A_{i}, A_{j}$ and $A_{k}, A_{l}$ represent the reactants and products of the chemical reaction, with $(i, j, k, l) \in\{(1,2,3,4),(2,1,4,3)\}$ and $(i, j, k, l) \in\{(3,4,1,2),(4,3,2,1)\}$ for the forward and backward chemical reactions respectively, the conservation laws of momentum and total energy (kinetic energy, internal energies and chemical binding energy) for reactive collisions are given by

$$
\begin{aligned}
m_{i} v_{i}+m_{j} v_{j} & =m_{k} v_{k}^{\prime}+m_{l} v_{l}^{\prime} \\
\frac{1}{2} m_{i}\left|v_{i}\right|^{2}+I_{i}+E_{i}+\frac{1}{2} m_{j}\left|v_{j}\right|^{2}+I_{j}+E_{j} & =\frac{1}{2} m_{k}\left|v_{k}^{\prime}\right|^{2}+I_{k}^{\prime}+E_{k}+\frac{1}{2} m_{l}\left|v_{l}^{\prime}\right|^{2}+I_{l}^{\prime}+E_{l} .
\end{aligned}
$$

Note that Equation (22) can be written in an equivalent form as

$$
\frac{1}{2} m_{i}\left|v_{i}\right|^{2}+I_{i}+\frac{1}{2} m_{j}\left|v_{j}\right|^{2}+I_{j}-\frac{E}{2}=\frac{1}{2} m_{k}\left|v_{k}^{\prime}\right|^{2}+I_{k}^{\prime}+\frac{1}{2} m_{l}\left|v_{l}^{\prime}\right|^{2}+I_{l}^{\prime}+\frac{E}{2}
$$

where $E$ has been defined in (18).

The post-collisional velocities can be expressed in terms of pre-collisional velocities and the corresponding expressions are derived from the conservation laws for reactive collisions (21), (22) and for non-reactive collisions (19), (20). They are deduced by applying the Borgnakke-Larsen procedure [9], which distributes the total energy of the colliding pair into kinetic and internal energies, when the collisions are non-reactive, or into kinetic, internal and chemical binding energies, when the collisions are reactive.

If we denote with $\mu_{i j}=m_{i} m_{j} /\left(m_{i}+m_{j}\right)$ the reduced mass of the colliding pair and introduce the two Borgnakke-Larsen parameters $R, r \in[0,1]$, we have, in the case of non-reactive elastic collisions,

$$
\begin{aligned}
& v_{i}^{\prime}=\frac{m_{i} v_{i}+m_{j} v_{j}}{m_{i}+m_{j}}+\frac{m_{j}}{m_{i}+m_{j}} \sqrt{\frac{2 R \mathscr{E}}{\mu_{i j}}} T_{\omega}\left[\frac{v_{i}-v_{j}}{\left|v_{i}-v_{j}\right|}\right] \\
& v_{j}^{\prime}=\frac{m_{i} v_{i}+m_{j} v_{j}}{m_{i}+m_{j}}-\frac{m_{i}}{m_{i}+m_{j}} \sqrt{\frac{2 R \mathscr{E}}{\mu_{i j}}} T_{\omega}\left[\frac{v_{i}-v_{j}}{\left|v_{i}-v_{j}\right|}\right],
\end{aligned}
$$

where $\mathscr{E}=\left(\mu_{i j}\left|v_{i}-v_{j}\right|^{2} / 2+I_{i}+I_{j}\right)=\left(\mu_{i j}\left|v_{i}^{\prime}-v_{j}^{\prime}\right|^{2} / 2+I_{i}^{\prime}+I_{j}^{\prime}\right)$ is the energy in the center of mass reference frame, $\omega \in \mathbb{S}^{2}$ is a unit vector and $T_{\omega}[x]=x-2(\omega \cdot x) \omega$ is the symmetry with respect to the plane $\{\omega\}^{\perp}$.

Suppose now that the collisions induce a forward chemical reaction and denote by $\mathscr{E}^{*}$ the total energy of the colliding pair. In this case, the conservation laws (21) and (22), give the post-collisional velocities, parameterized by a unit vector $\omega \in \mathbb{S}^{2}$ :

$$
\begin{aligned}
& v_{3}^{\prime}=\frac{m_{1} v_{1}+m_{2} v_{2}}{m_{1}+m_{2}}+\frac{m_{4}}{m_{3}+m_{4}} \sqrt{\frac{2}{\mu_{34}}}\left(R \mathscr{E}^{*}-\frac{E}{6}\right)^{1 / 2} T_{\omega}\left[\frac{v_{1}-v_{2}}{\left|v_{1}-v_{2}\right|}\right] \\
& v_{4}^{\prime}=\frac{m_{1} v_{1}+m_{2} v_{2}}{m_{1}+m_{2}}-\frac{m_{3}}{m_{3}+m_{4}} \sqrt{\frac{2}{\mu_{34}}}\left(R \mathscr{E}^{*}-\frac{E}{6}\right)^{1 / 2} T_{\omega}\left[\frac{v_{1}-v_{2}}{\left|v_{1}-v_{2}\right|}\right] .
\end{aligned}
$$


The reactive post-collisional velocities for the backward reaction in terms of precollisional velocities are

$$
\begin{aligned}
& v_{1}^{\prime}=\frac{m_{3} v_{3}+m_{4} v_{4}}{m_{3}+m_{4}}+\frac{m_{2}}{m_{1}+m_{2}} \sqrt{\frac{2}{\mu_{12}}}\left(R \mathscr{E}^{*}+\frac{E}{6}\right)^{1 / 2} T_{\omega}\left[\frac{v_{3}-v_{4}}{\left|v_{3}-v_{4}\right|}\right] \\
& v_{2}^{\prime}=\frac{m_{3} v_{3}+m_{4} v_{4}}{m_{3}+m_{4}}-\frac{m_{1}}{m_{1}+m_{2}} \sqrt{\frac{2}{\mu_{12}}}\left(R \mathscr{E}^{*}+\frac{E}{6}\right)^{1 / 2} T_{\omega}\left[\frac{v_{3}-v_{4}}{\left|v_{3}-v_{4}\right|}\right] .
\end{aligned}
$$

The evolution of the distribution functions $f_{i}$, with $i=1, \ldots, 4$, is governed by the system of kinetic equations

$$
\frac{\partial f_{i}}{\partial t}+v \cdot \nabla_{x} f_{i}=\sum_{j=1}^{4} Q_{i j}^{e}\left(f_{i}, f_{j}\right)+Q_{i}^{\text {react }}, \quad i=1, \ldots, 4 .
$$

In the sum written above, when $i \neq j$, the notation $Q_{i j}^{e}$ represents the bi-species elastic operator associated to collisions between one particle of constituent $i$ and another one of constituent $j$. When $i=j$, the operator $Q_{i i}^{e}$ is nothing but the standard mono-species elastic collisional operator. Moreover, $Q_{i}^{\text {react }}$ represents the reactive collisional operator. The operators $Q_{i j}^{e}$ and $Q_{i}^{\text {react }}$ are defined as follows. For nonreactive interactions, the operators are

$$
\begin{aligned}
Q_{i j}^{e}\left(f_{i}, f_{j}\right)= & \int_{\mathbb{R}^{3}} \int_{0}^{+\infty} \int_{0}^{1} \int_{0}^{1} \int_{\mathbb{S}^{2}}\left[f_{i}\left(v_{i}^{\prime}, I_{i}^{\prime}\right) f_{j}\left(v_{j}^{\prime}, I_{j}^{\prime}\right)-f_{i}(v, I) f_{j}\left(v_{j}, I_{j}\right)\right] \\
& \times B_{i j}\left(v, v_{j}, I, I_{j}, R, r, \omega\right)(1-R)\left|v-v_{j}\right|^{-1} \varphi_{i}(I)^{-1} \mathrm{~d} \omega \mathrm{d} r \mathrm{~d} R \mathrm{~d} I_{j} \mathrm{~d} v_{j},
\end{aligned}
$$

where $B_{i j}$ are suitable cross sections and $v_{i}^{\prime}, v_{j}^{\prime}, I_{i}^{\prime}$ and $I_{j}^{\prime}$ are given by

$$
\begin{gathered}
v_{i}^{\prime}=\frac{m_{i} v+m_{j} v_{j}}{m_{i}+m_{j}}+\frac{m_{j}}{m_{i}+m_{j}} \sqrt{\frac{2 R \mathscr{E}}{\mu_{i j}}} T_{\omega}\left[\frac{v-v_{j}}{\left|v-v_{j}\right|}\right], \\
v_{j}^{\prime}=\frac{m_{i} v+m_{j} v_{j}}{m_{i}+m_{j}}-\frac{m_{i}}{m_{i}+m_{j}} \sqrt{\frac{2 R \mathscr{E}}{\mu_{i j}}} T_{\omega}\left[\frac{v-v_{j}}{\left|v-v_{j}\right|}\right], \\
I_{i}^{\prime}=\left(\frac{\mu_{i j}}{2}\left|v-v_{j}\right|^{2}+I+I_{j}\right) r(1-R), I_{j}^{\prime}=\left(\frac{\mu_{i j}}{2}\left|v-v_{j}\right|^{2}+I+I_{j}\right)(1-r)(1-R) .
\end{gathered}
$$

The reactive collisional operators are more involved. Let $B^{\text {react }}: \mathbb{R}^{3} \times \mathbb{R}^{3} \times \mathbb{R}^{+} \times$ $\mathbb{R}^{+} \times[0,1]^{2} \times \mathbb{S}^{2} \rightarrow \mathbb{R}^{+}$be a suitable cross-section.

In the case of the forward reaction, we define the total energy as

$$
\mathscr{E}_{1}^{*}=\frac{1}{2} \mu_{12}\left|v-v_{2}\right|^{2}+I+I_{2}-\frac{E}{2} .
$$

Then, the reactive post-collisional velocities and internal energies can be written in terms of the pre-collisional quantities in the following way: 


$$
\begin{gathered}
v_{3}^{\prime}=\frac{m_{1} v+m_{2} v_{2}}{m_{1}+m_{2}}+\frac{m_{4}}{m_{3}+m_{4}} \sqrt{\frac{2}{\mu_{34}}}\left(R \mathscr{E}_{1}^{*}-\frac{E}{6}\right)^{1 / 2} T_{\omega}\left[\frac{v-v_{2}}{\left|v-v_{2}\right|}\right], \\
v_{4}^{\prime}=\frac{m_{1} v+m_{2} v_{2}}{m_{1}+m_{2}}-\frac{m_{3}}{m_{3}+m_{4}} \sqrt{\frac{2}{\mu_{34}}}\left(R \mathscr{E}_{1}^{*}-\frac{E}{6}\right)^{1 / 2} T_{\omega}\left[\frac{v-v_{2}}{\left|v-v_{2}\right|}\right], \\
I_{3}^{\prime}=r(1-R) \mathscr{E}_{1}^{*}-\frac{E}{6}, \quad I_{4}^{\prime}=(1-r)(1-R) \mathscr{E}_{1}^{*}-\frac{E}{6} .
\end{gathered}
$$

Consider the set

$$
\begin{aligned}
& F_{1}=\left\{\left(I, I_{2}, r, R, v, v_{2}\right): I \geq \frac{E}{6}, I_{2} \geq \frac{E}{6}, R \mathscr{E}_{1}^{*} \geq \frac{E}{6},\right. \\
& \left.\frac{\mu_{12}}{2}\left|v-v_{2}\right|^{2} \geq \frac{E}{6},(1-R) r \mathscr{E}_{1}^{*} \geq \frac{E}{6},(1-R)(1-r) \mathscr{E}_{1}^{*} \geq \frac{E}{6} .\right\} .
\end{aligned}
$$

Then, the collisional integral describing the forward chemical reaction is

$$
\begin{gathered}
Q_{1}^{\text {react }}(v, I)=\int_{\mathbb{R}^{3}} \int_{0}^{+\infty} \int_{0}^{1} \int_{0}^{1} \int_{\mathbb{S}^{2}}\left[\left(\frac{m_{1} m_{2}}{m_{3} m_{4}}\right)^{3} f_{3}\left(v_{3}^{\prime}, I_{3}^{\prime}\right) f_{4}\left(v_{4}^{\prime}, I_{4}^{\prime}\right)-f_{1}(v, I) f_{2}\left(v_{2}, I_{2}\right)\right] \\
\times \mathbb{1}_{\xi \in F_{1}} B^{\text {react }}\left(v, v_{2}, I, I_{2}, R, r, \omega\right) \frac{(1-R)}{\left(m_{1} m_{2}\right)^{2}\left|v-v_{2}\right| \varphi_{1}(I)} \mathrm{d} \omega \mathrm{d} r \mathrm{~d} R \mathrm{~d} I_{2} \mathrm{~d} v_{2} .
\end{gathered}
$$

The structure of $Q_{2}^{\text {react }}$ is similar. We define the total energy

$$
\mathscr{E}_{2}^{*}=\frac{1}{2} \mu_{12}\left|v-v_{1}\right|^{2}+I+I_{1}-\frac{E}{2},
$$

the reactive post-collisional velocities and internal energies

$$
\begin{aligned}
& v_{3}^{\prime}=\frac{m_{1} v_{1}+m_{2} v}{m_{1}+m_{2}}+\frac{m_{4}}{m_{3}+m_{4}} \sqrt{\frac{2}{\mu_{34}}}\left(R \mathscr{E}_{2}^{*}-\frac{E}{6}\right)^{1 / 2} T_{\omega}\left[\frac{v_{1}-v}{\left|v_{1}-v\right|}\right], \\
& v_{4}^{\prime}=\frac{m_{1} v_{1}+m_{2} v}{m_{1}+m_{2}}-\frac{m_{3}}{m_{3}+m_{4}} \sqrt{\frac{2}{\mu_{34}}}\left(R \mathscr{E}_{2}^{*}-\frac{E}{6}\right)^{1 / 2} T_{\omega}\left[\frac{v_{1}-v}{\left|v_{1}-v\right|}\right], \\
& I_{3}^{\prime}=r(1-R) \mathscr{E}_{2}^{*}-\frac{E}{6}, \quad I_{4}^{\prime}=(1-r)(1-R) \mathscr{E}_{2}^{*}-\frac{E}{6},
\end{aligned}
$$

and the set

$$
\begin{aligned}
& F_{2}=\left\{\left(I, I_{1}, r, R, v, v_{1}\right): I \geq \frac{E}{6}, I_{1} \geq \frac{E}{6}, R \mathscr{E}_{2}^{*} \geq \frac{E}{6},\right. \\
& \left.\frac{\mu_{12}}{2}\left|v-v_{1}\right|^{2} \geq \frac{E}{6},(1-R) r \mathscr{E}_{2}^{*} \geq \frac{E}{6},(1-R)(1-r) \mathscr{E}_{2}^{*} \geq \frac{E}{6}\right\} .
\end{aligned}
$$


The collisional integral $Q_{2}^{\text {react }}$ describing the forward chemical reaction is hence

$$
\begin{gathered}
Q_{2}^{\text {react }}(v, I)=\int_{\mathbb{R}^{3}} \int_{0}^{+\infty} \int_{0}^{1} \int_{0}^{1} \int_{\mathbb{S}^{2}}\left[\left(\frac{m_{1} m_{2}}{m_{3} m_{4}}\right)^{3} f_{3}\left(v_{3}^{\prime}, I_{3}^{\prime}\right) f_{4}\left(v_{4}^{\prime}, I_{4}^{\prime}\right)-f_{2}(v, I) f_{1}\left(v_{1}, I_{1}\right)\right] \\
\times \mathbb{1}_{\xi \in F_{2}} B^{\text {react }}\left(v, v_{1}, I, I_{1}, R, r, \omega\right) \frac{(1-R)}{\left(m_{1} m_{2}\right)^{2}\left|v-v_{1}\right| \varphi_{2}(I)} \mathrm{d} \omega \mathrm{d} r \mathrm{~d} R \mathrm{~d} I_{1} \mathrm{~d} v_{1} .
\end{gathered}
$$

In the case of the backward reaction we treat two collisional terms. Denote

$$
\mathscr{E}_{3}^{*}=\frac{1}{2} \mu_{34}\left|v-v_{4}\right|^{2}+I+I_{4}+\frac{E}{2}
$$

the total energy. We can write the reactive post collisional velocities and internal energies as follows:

$$
\begin{aligned}
& v_{1}^{\prime}=\frac{m_{3} v+m_{4} v_{4}}{m_{3}+m_{4}}+\frac{m_{2}}{m_{1}+m_{2}} \sqrt{\frac{2}{\mu_{12}}}\left(R \mathscr{E}_{3}^{*}+\frac{E}{6}\right)^{1 / 2} T_{\omega}\left[\frac{v-v_{4}}{\left|v-v_{4}\right|}\right], \\
& v_{2}^{\prime}=\frac{m_{3} v+m_{4} v_{4}}{m_{3}+m_{4}}-\frac{m_{1}}{m_{1}+m_{2}} \sqrt{\frac{2}{\mu_{12}}}\left(R \mathscr{E}_{3}^{*}+\frac{E}{6}\right)^{1 / 2} T_{\omega}\left[\frac{v-v_{4}}{\left|v-v_{4}\right|}\right], \\
& I_{1}^{\prime}=r(1-R) \mathscr{E}_{3}^{*}+\frac{E}{6}, \quad I_{2}^{\prime}=(1-r)(1-R) \mathscr{E}_{3}^{*}+\frac{E}{6} .
\end{aligned}
$$

The admissible set $F_{3}$ is

$$
F_{3}=\left\{\left(I_{4}, r, R, v_{4}\right): I_{4} \geq 0, v_{4} \in \mathbb{R}^{3}, r, R \in[0,1]\right\} .
$$

The collisional integral $Q_{3}^{\text {react }}$ describing the backward reaction is defined by

$$
\begin{gathered}
Q_{3}^{\text {react }}(v, I)=\int_{\mathbb{R}^{3}} \int_{0}^{+\infty} \int_{0}^{1} \int_{0}^{1} \int_{\mathbb{S}^{2}}\left[\left(\frac{m_{3} m_{4}}{m_{1} m_{2}}\right)^{3} f_{1}\left(v_{1}^{\prime}, I_{1}^{\prime}\right) f_{2}\left(v_{2}^{\prime}, I_{2}^{\prime}\right)-f_{3}(v, I) f_{4}\left(v_{4}, I_{4}\right)\right] \\
\times \mathbb{1}_{\xi \in F_{3}} B^{\text {react }}\left(v, v_{4}, I, I_{4}, R, r, \omega\right) \frac{(1-R)}{\left(m_{3} m_{4}\right)^{2}\left|v-v_{4}\right| \varphi_{3}(I)} \mathrm{d} \omega \mathrm{d} r \mathrm{~d} R \mathrm{~d} I_{4} \mathrm{~d} v_{4} .
\end{gathered}
$$

Finally, let

$$
\mathscr{E}_{4}^{*}=\frac{1}{2} \mu_{34}\left|v-v_{3}\right|^{2}+I+I_{3}+\frac{E}{2}
$$

be the total energy of the binary encounter. Then, we can write the reactive postcollisional velocities and internal energies in the following way:

$$
v_{1}^{\prime}=\frac{m_{3} v_{3}+m_{4} v}{m_{3}+m_{4}}+\frac{m_{2}}{m_{1}+m_{2}} \sqrt{\frac{2}{\mu_{12}}}\left(R \mathscr{E}_{4}^{*}+\frac{E}{6}\right)^{1 / 2} T_{\omega}\left[\frac{v_{3}-v}{\left|v_{3}-v\right|}\right],
$$




$$
\begin{gathered}
v_{2}^{\prime}=\frac{m_{3} v_{3}+m_{4} v}{m_{3}+m_{4}}-\frac{m_{1}}{m_{1}+m_{2}} \sqrt{\frac{2}{\mu_{12}}}\left(R \mathscr{E}_{4}^{*}+\frac{E}{6}\right)^{1 / 2} T_{\omega}\left[\frac{v_{3}-v}{\left|v_{3}-v\right|}\right] . \\
I_{1}^{\prime}=r(1-R) \mathscr{E}_{4}^{*}+\frac{E}{6}, \quad I_{2}^{\prime}=(1-r)(1-R) \mathscr{E}_{4}^{*}+\frac{E}{6} .
\end{gathered}
$$

The admissible set $F_{4}$ is

$$
F_{4}=\left\{\left(I_{3}, r, R, v_{3}\right): I_{3} \geq 0, \quad v_{3} \in \mathbb{R}^{3}, r, R \in[0,1]\right\},
$$

and the reactive collisional integral $Q_{4}^{\text {react }}$, describing the backward reaction, is defined by

$$
\begin{gathered}
Q_{4}^{\text {react }}(v, I)=\int_{\mathbb{R}^{3}} \int_{0}^{+\infty} \int_{0}^{1} \int_{0}^{1} \int_{\mathbb{S}^{2}}\left[\left(\frac{m_{3} m_{4}}{m_{1} m_{2}}\right)^{3} f_{1}\left(v_{1}^{\prime}, I_{1}^{\prime}\right) f_{2}\left(v_{2}^{\prime}, I_{2}^{\prime}\right)-f_{4}(v, I) f_{3}\left(v_{3}, I_{3}\right)\right] \\
\times \mathbb{1}_{\xi \in F_{4}} B^{\text {react }}\left(v, v_{3}, I, I_{3}, R, r, \omega\right) \frac{(1-R)}{\left(m_{3} m_{4}\right)^{2}\left|v-v_{3}\right| \varphi_{4}(I)} \mathrm{d} \omega \mathrm{d} r \mathrm{~d} R \mathrm{~d} I_{3} \mathrm{~d} v_{3} .
\end{gathered}
$$

In [16], the equilibria for the kinetic system (23) have been studied in two steps, first by assuming that the mechanical equilibrium is reached, and then by considering the chemical equilibrium associated to the reactive collisional operator.

These equilibria are again called Maxwellians, because they play the same role as the Maxwellian functions of the classical Boltzmann equation. Let $c_{i} \geq 0, i=$ $1, \ldots, 4, u \in \mathbb{R}^{3}$ and $T>0$. Then, the reactive Maxwellians of the model have the form

$$
\mathscr{M}\left(c_{i}, u, T\right)=\frac{c_{i}(t, x)}{q_{i}(T(t, x))}\left(\frac{m_{i}}{2 \pi k_{B} T(t, x)}\right)^{3 / 2} \exp \left(-\frac{m_{i}|v-u(t, x)|^{2}}{2 k_{B} T(t, x)}-\frac{I}{k_{B} T(t, x)}\right)
$$

where

$$
q_{i}(T(t, x))=\int_{0}^{+\infty} \varphi_{i}(I) \exp \left(-\frac{I}{k_{B} T(t, x)}\right) \mathrm{d} I
$$

can be interpreted as a Laplace transform of $\varphi_{i}$ for all $i$.

Note that this model may describe a non-reactive mixture of polyatomic gases, by simply neglecting the reactive collision kernels. In this case, it can be viewed as a generalization of [14], which studied non-reactive polyatomic gaseous mixtures.

\section{The Maxwell-Stefan diffusion limit for non-reactive Boltzmann systems}

In the previous section, we have described two kinetic models for reactive and nonreactive mixtures. The diffusive limit of these systems is based on the study of the 
asymptotic behaviour, as the mean free path tends to zero, of the rescaled kinetic system in the diffusive scaling, by supposing that the solutions are perturbations of the local Maxwellian state. It is indeed clear that, if the initial conditions are local Maxwellians, there are no reasons which guarantee that the Maxwellian structure is kept by the time evolution. However, it is possible to prove that the solutions of the system stay close to Maxwellian functions, with a reminder which is small as the mean free path tends to zero.

We first describe the formal asymptotics, then we consider the rigorous derivation of the diffusive system.

\subsection{The formal asymptotics}

In [13], Boudin, Grec, and Salvarani study the Boltzmann system described in Section 3, in a spatial domain $\Omega$, in the case of Maxwellian molecules, i.e. by supposing that each cross section $B_{i j}$ depends on $v, v_{*}$ and $\sigma$ only through the deviation angle $\theta \in[0, \pi]$ between $\left(v-v_{*}\right)$ and $\sigma$. This means that, for each $(i, j)$, there exists a function $b_{i j}:[-1,1] \rightarrow \mathbb{R}^{+}$such that

$$
B_{i j}\left(v, v_{*}, \sigma\right)=b_{i j}\left(\frac{v-v_{*}}{\left|v-v_{*}\right|} \cdot \sigma\right)=b_{i j}(\cos \theta) .
$$

In the article is moreover supposed that $b_{i j}$ is even and that $b_{i j} \in L^{1}(-1,1)$ (Grad's angular cutoff assumption).

After introducing the mean free path $\varepsilon>0$, the authors wrote the kinetic system in the diffusive scaling. By denoting with $\left(f_{i}^{\mathcal{E}}\right)_{1 \leq i \leq \mathscr{I}}$ the corresponding unknowns in this regime, each distribution function $f_{i}^{\mathcal{E}}$ solves

$$
\varepsilon \partial_{t} f_{i}^{\varepsilon}+v \cdot \nabla_{x} f_{i}^{\varepsilon}=\frac{1}{\varepsilon} \sum_{j=1}^{\mathscr{I}} Q_{i j}\left(f_{i}^{\varepsilon}, f_{j}^{\varepsilon}\right), \quad \text { on } \mathbb{R}_{*}^{+} \times \Omega \times \mathbb{R}^{3} .
$$

The analysis has been carried out in the isothermal case, i.e. by supposing that the temperature $T$ is constant with respect to the time variable and homogeneous with respect to the spatial variable. Once introduced the quantities

$$
c_{i}^{\varepsilon}: \mathbb{R}^{+} \times \Omega \rightarrow \mathbb{R}^{+}, \quad u_{i}^{\varepsilon}: \mathbb{R}^{+} \times \Omega \rightarrow \mathbb{R}^{3}, \quad 1 \leq i \leq \mathscr{I},
$$

the article assumes that the system evolution leaves the distribution functions, up to a correction of lower order, in the local Maxwellian state,

$f_{i}^{\mathcal{E}}=c_{i}^{\mathcal{\varepsilon}}(t, x)\left(\frac{m_{i}}{2 \pi k_{B} T}\right)^{3 / 2} e^{-m_{i}\left|v-\varepsilon u_{i}^{\varepsilon}(t, x)\right|^{2} / 2 k_{B} T}(1+\mathscr{O}(\varepsilon)), t>0,(x, v) \in \Omega \times \mathbb{R}^{3}$,

where the quantity $\varepsilon u_{i}^{\varepsilon}$ is the bulk velocity, of order $\varepsilon$, whose precise form may depend on the considered species of the mixture. By supposing that all the initial 
quantities

$$
c_{i}^{\text {in }}: \Omega \rightarrow \mathbb{R}^{+}, \quad u_{i}^{\text {in }}: \Omega \rightarrow \mathbb{R}^{3}, \quad 1 \leq i \leq \mathscr{I},
$$

do not depend on $\varepsilon$ and that

$$
\sum_{i=1}^{\mathscr{I}} c_{i}^{\text {in }}=1 \quad \text { on } \Omega
$$

the authors prove that, in the limit $\varepsilon \rightarrow 0$, for $(t, x) \in \mathbb{R}^{+} \times \Omega$, by supposing the existence of the limits

$$
c_{i}(t, x)=\lim _{\varepsilon \rightarrow 0^{+}} c_{i}^{\varepsilon}(t, x), \quad J_{i}(t, x)=\lim _{\varepsilon \rightarrow 0^{+}} c_{i}^{\varepsilon}(t, x) u_{i}^{\varepsilon}(t, x)
$$

and that

$$
c:=\sum_{i=1}^{\mathscr{I}} c_{i}=\sum_{i=1}^{\mathscr{I}} c_{i}^{\mathrm{in}}=1 \quad \text { on } \Omega,
$$

the pairs $\left(c_{i}, J_{i}\right)$ formally satisfy the Maxwell-Stefan diffusion system (1) and give an explicit form of the binary diffusion coefficients:

$$
k_{i j}=\frac{2 \pi m_{i} m_{j}\left\|b_{i j}\right\|_{L^{1}}}{\left(m_{i}+m_{j}\right) k_{B} T} .
$$

These binary diffusion coefficients are symmetric $\left(k_{i j}=k_{j i}\right.$ for all $\left.i, j=1, \ldots, \mathscr{I}\right)$ and depend on the cross-section (in this case, the cross-section of Maxwell molecules with Grad's angular cutoff).

The authors have not deduced, in the limiting procedure, the equimolar closure relationship (2). However, they have proved that, formally,

$$
\nabla_{x} \cdot\left(\sum_{i=1}^{\mathscr{I}} J_{i}\right)=0 .
$$

Other cross sections give different forms of the binary diffusion coefficients. In particular, Hutridurga and Salvarani [23] derived the expression of the binary diffusion coefficients under suitable assumptions on the collision kernels $B_{i j}$. They suppose that the collision kernels $B_{i j}$ depend on the modulus of the relative velocity $\left|v-v_{*}\right|$ and on the cosine of the deviation angle $\theta$, i.e.

$$
B_{i j}\left(v, v_{*}, \sigma\right)=\Phi\left(\left|v-v_{*}\right|\right) b_{i j}(\cos \theta) .
$$

Moreover, they assume that the angular collision kernels $b_{i j}$ belong to the class $L^{1}(-1,+1)$ and that they are even functions with respect to their argument. Furthermore, they suppose that the kinetic collision kernel $\Phi\left(\left|v-v_{*}\right|\right)$ is analytic in the following sense: there exists a family $\left\{a_{n}\right\}_{n \in \mathbb{N}^{*}} \subset \mathbb{R}$ such that $\Phi$ can be written as a uniformly converging even power series: 


$$
\Phi\left(\left|v-v_{*}\right|\right)=\sum_{n \in \mathbb{N}^{*}} a_{n}\left|v-v_{*}\right|^{2 n} .
$$

Under these hypotheses, the binary diffusion coefficients computed in [23] have the form

$$
\begin{gathered}
k_{i j}=\left\{a_{0} \frac{2 \pi m_{i} m_{j}\left\|b_{i j}\right\|_{L^{1}}}{\left(m_{i}+m_{j}\right) k_{B} T}+a_{1} 10 \pi\left\|b_{i j}\right\|_{L^{1}}+\right. \\
\sum_{n \geq 2} a_{n} \frac{2 \pi\left\|b_{i j}\right\|_{L^{1}}\left(m_{i} m_{j}\right)}{\left(m_{i}+m_{j}\right) k_{B} T} \sum_{n_{1}+n_{2}+n_{3}=n} \frac{n !}{n_{1} ! n_{2} ! n_{3} !} \sum_{\substack{\alpha, \beta, \gamma, \delta, \rho, \eta \in 2 \mathbb{N}^{*} \\
\alpha+\beta=2 n_{1} \\
\gamma+\delta=2 n_{2} \\
\rho+\eta=2 n_{3}}} \frac{\left(2 n_{1}\right) !}{\alpha ! \beta !} \frac{\left(2 n_{2}\right) !}{\gamma ! \delta !} \frac{\left(2 n_{3}\right) !}{\rho ! \eta !} \\
\left.\left.\times \mathscr{E}(\alpha, \beta, \gamma, \delta, \rho, \eta)\left(\frac{k_{B} T}{m_{i}}\right)^{(\alpha+\gamma+\rho) / 2}\left(\frac{k_{B} T}{m_{j}}\right)^{(\beta+\delta+\eta) / 2}\right)\right\},
\end{gathered}
$$

where

$$
\begin{array}{r}
\mathscr{E}(\alpha, \beta, \gamma, \delta, \rho, \eta)=((\alpha-1)(\alpha-3) \cdots 1)((\beta-1)(\beta-3) \cdots 1)((\gamma-1)(\gamma-3) \cdots 1) \\
\times((\delta-1)(\delta-3) \cdots 1)((\rho-1)(\rho-3) \cdots 1)((\eta-1)(\eta-3) \cdots 1) .
\end{array}
$$

The binary diffusion coefficients depend on the reduced mass of the species, on the temperature and on the cross sections of the kinetic model. Therefore, by tuning the coefficients $a_{n}$ in the kinetic cross section (27), it is possible to obtain several forms of temperature dependency for the binary diffusion coefficients and compare them with the experiments. When $a_{i}=0$ for all $i \geq 1$, the formula written above corresponds to the binary diffusion coefficients already deduced in Equation (26).

A gas of hard spheres of fixed diameter is not included in the previous analysis. The study of this case has been carried out by Anwasia [1].

In [11], Boudin, Grec and Pavan provide another approach for going beyond Maxwellian molecules. They propose a semi-explicit link between the MaxwellStefan diffusion coefficients and the cross sections of the corresponding rescaled Boltzmann system for monatomic gaseous mixtures in the diffusive scaling, for general cross sections $B_{i j}=B_{i j}\left(v, v_{*}, \sigma\right)$ satisfying the Galilean invariance and the microreversibility assumptions, under Grad's angular cut-off hypothesis. They prove that

$$
\begin{aligned}
k_{i j}= & \iiint B_{i j}\left(v, v_{*}, \sigma\right) \exp \left[-\frac{m_{i}}{2 \pi k_{B} T}|v|^{2}-\frac{m_{j}}{2 \pi k_{B} T}\left|v_{*}\right|^{2}\right]\left[m_{i}\left(v^{\prime}-v\right)\right]^{2} \mathrm{~d} \sigma, \mathrm{d} v_{*} \mathrm{~d} v \\
& =\frac{m_{i} m_{j}}{6\left(k_{B} T\right)^{2}\left(m_{i}+m_{j}\right)}\left(\frac{m_{i}}{2 \pi k_{B} T}\right)^{3 / 2}\left(\frac{m_{j}}{2 \pi k_{B} T}\right)^{3 / 2} \iiint B_{i j}\left(v, v_{*}, \sigma\right) \times
\end{aligned}
$$




$$
\exp \left[-\frac{m_{i}}{2 \pi k_{B} T}|v|^{2}-\frac{m_{j}}{2 \pi k_{B} T}\left|v_{*}\right|^{2}\right]\left(v-v_{*}+\left|v^{\prime}-v\right| \sigma\right) \cdot\left(m_{i} v-m_{j} v_{*}\right) \mathrm{d} \sigma, \mathrm{d} v_{*} \mathrm{~d} v \text {. }
$$

These two equivalent forms of the binary diffusion coefficients show their positivity as well as their symmetry.

We moreover quote [22], which derived, by using the same approach, a system of Maxwell-Stefan type when the temperature is not uniform in space nor constant in time. In the diffusive limit, the authors show that the kinetic temperature of each species of the mixture tends to the same temperature function $T=T(t, x),(t, x) \in$ $(0, \infty) \times \Omega, \Omega \subseteq \mathbb{R}^{3}$ as $\varepsilon \rightarrow 0$. The structure of the system they derived is:

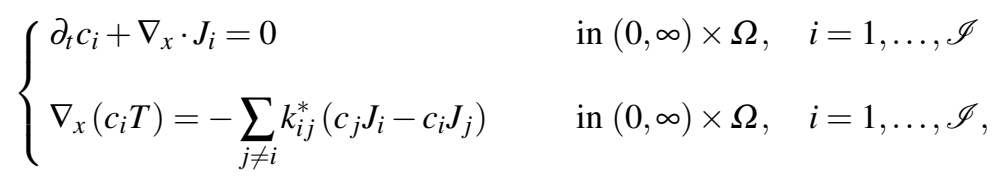

The binary diffusion coefficients $k_{i j}^{*}$, as in the previously described articles, depend on the cross sections. In particular, if the hypotheses on the cross sections are the same as in [1], [11], [13] or [23] respectively, then the binary diffusion coefficients in (28) are of the form $k_{i j}^{*}=T(t, x) k_{i j}$, where $k_{i j}$ are the binary diffusion coefficients described in [1], [11], [13] or [23] .

\subsection{The rigorous diffusive asymptotics}

The article by Bondesan and Briant [8] studies the Maxwell-Stefan asymptotics, in the 3-dimensional torus $\mathbb{T}^{3}$, of the system of coupled Boltzmann equations for mixtures (5)-(6), with collisional integrals of the form (8)-(9) and suitable cross sections (of cutoff Maxwellian type, or hard potentials, or hard spheres). The starting point is the Cauchy theory, proved by the same authors in [7], whose precise result is the following.

Theorem 1. Let $s>3$ be an integer, $\bar{u}: \mathbb{R}^{+} \times \mathbb{T}^{3} \rightarrow \mathbb{R}^{3}$ be in $L^{\infty}\left(\mathbb{R}^{+} ; H^{s}\left(\mathbb{T}^{3}\right)\right)$ with $\nabla_{x} \cdot \bar{u}=0$, and consider $\overline{\mathbf{c}}>0$. Suppose that there exist $\delta_{M S}, C_{M S}, C_{M S}^{\prime}, \lambda_{M S}>0$ such that for all $\varepsilon \in(0,1]$ and for any initial datum $\left(\tilde{\mathbf{c}}^{\text {in }}, \tilde{\mathbf{u}}^{\text {in }}\right) \in H^{s}\left(\mathbb{T}^{3}\right) \times H^{s-1}\left(\mathbb{T}^{3}\right)$, for almost any $x \in \mathbb{T}^{3}$ and for any $1 \leq i \leq \mathscr{I}$, the following requirement are satisfied:

i) Mass compatibility: $\sum_{i=1}^{\mathscr{I}} \tilde{c}_{i}^{\mathrm{in}}(x)=0$ and $\int_{\mathbb{T}^{3}} \tilde{c}_{i}^{\text {in }}(x) \mathrm{d} x=0$,

ii) Mass positivity: $\bar{c}_{i}+\varepsilon \tilde{c}_{i}^{\text {in }}(x)>0$,

iii)Moment compatibility: $\nabla_{x} \tilde{c}_{i}^{\text {in }}=\sum_{j \neq i} k_{i j} c_{i}^{\text {in }} c_{j}^{\text {in }}\left(\tilde{u}_{j}^{\text {in }}-\tilde{u}_{i}^{\text {in }}\right)$,

iv) Smallness assumptions: $\left\|\tilde{\mathbf{c}}^{\text {in }}\right\|_{H_{x}^{s}} \leq \delta_{M S}$ and $\|\bar{u}\|_{L_{t}^{\infty} H_{x}^{s}} \leq \delta_{M S}$.

Then, there exists a unique weak solution 


$$
(\mathbf{c}, \mathbf{u})=(\overline{\mathbf{c}}+\varepsilon \tilde{\mathbf{c}}, \overline{\mathbf{u}}+\varepsilon \tilde{\mathbf{u}})
$$

in $L^{\infty}\left(\mathbb{R}^{+} ; H^{s}\left(\mathbb{T}^{3}\right)\right) \times L^{\infty}\left(\mathbb{R}^{+} ; H^{s-1}\left(\mathbb{T}^{3}\right)\right)$ to the incompressible Maxwell-Stefan system

$$
\begin{gathered}
\partial_{t} c_{i}+\nabla_{x} \cdot\left(c_{i} u_{i}\right)=0, \\
-\nabla_{x} c_{i}=\sum_{j=1}^{\mathscr{I}} k_{i j} c_{i} c_{j}\left(u_{i}-u_{j}\right), \\
\nabla_{x} \cdot\left(\sum_{i=1}^{\mathscr{I}} c_{i} u_{i}\right)=0,
\end{gathered}
$$

such that initially $\left.(\tilde{\mathbf{c}}, \tilde{\mathbf{u}})\right|_{t=0}=\left(\tilde{\mathbf{c}}^{\text {in }}, \tilde{\mathbf{u}}^{\text {in }}\right)$ a.e. on $\mathbb{T}^{3}$. In particular, if $s>4$ and $\bar{u} \in C^{0}\left(\mathbb{R}^{+} ; H^{s}\left(\mathbb{T}^{3}\right)\right)$, then the pair $(\mathbf{c}, \mathbf{u})$ also belongs to $C^{0}\left(\mathbb{R}^{+} ; H^{s-1}\left(\mathbb{T}^{3}\right)\right) \times$ $C^{0}\left(\mathbb{R}^{+} ; H^{s-2}\left(\mathbb{T}^{3}\right)\right)$. Moreover, $\mathbf{c}$ is positive and the following relations hold a.e. on $\mathbb{R}^{+} \times \mathbb{T}^{3}$ :

$$
\langle\mathbf{c}, \tilde{\mathbf{u}}\rangle=\sum_{i=1}^{\mathscr{F}} c_{i}(t, x) \tilde{u}_{i}(t, x)=0 \quad \text { and } \quad \int_{\mathbb{T}^{3}} \tilde{c}_{i}(t, x) \mathrm{d} x=0 .
$$

Finally, for almost any time $t \geq 0$

$$
\begin{array}{r}
\|\tilde{\mathbf{c}}\|_{H_{x}^{s}\left(\overline{\mathbf{c}}^{-\frac{1}{2}}\right)} \leq e^{-t \lambda_{M S}}\left\|\tilde{\mathbf{c}}^{\mathrm{in}}\right\|_{H_{x}^{s}\left(\overline{\mathbf{c}}^{-\frac{1}{2}}\right)}, \quad\|\tilde{\mathbf{u}}\|_{H_{x}^{s-1}} \leq C_{M S} e^{-t \lambda_{M S}}\left\|\tilde{\mathbf{c}}^{\mathrm{in}}\right\|_{H_{x}^{s}\left(\overline{\mathbf{c}}^{-\frac{1}{2}}\right)}, \\
\int_{0}^{t} e^{2(t-\tau) \lambda_{M S}}\|\tilde{\mathbf{u}}(\tau)\|_{H_{x}^{s}}^{2} \mathrm{~d} \tau \leq C_{M S}^{\prime}\left\|\tilde{\mathbf{c}}^{\mathrm{in}}\right\|_{H_{x}^{s}\left(\overline{\mathbf{c}}^{-\frac{1}{2}}\right)}^{2} .
\end{array}
$$

The constants $\delta_{M S}, \lambda_{M S}, C_{M S}$ and $C_{M S}^{\prime}$ are constructive and only depend on s, the number of species $\mathscr{I}$, the diffusion coefficients $\left(k_{i j}\right)_{1 \leq i, j \leq \mathscr{I}}$ and the constant vector $\overline{\mathbf{c}}$. In particular, they are independent of the parameter $\varepsilon$.

This theorem guarantees the possibility of building a Cauchy theory around the macroscopic state $(\overline{\mathbf{c}}, \overline{\mathbf{u}})$, instead of considering the equilibrium $(\overline{\mathbf{c}}, \mathbf{0})$. It is hence possible to give a complete description of systems having an equilibrium state with a constant mass vector $\overline{\mathbf{c}}$. Actually, this theorem does not provide strong uniqueness for the solutions, because infinitely many solutions to the Maxwell-Stefan system can be constructed by considering different constant $\overline{\mathbf{c}}$ and $\overline{\mathbf{u}}$ satisfying the incompressible condition. However, as soon as it is possible to fix a macroscopic equilibrium $(\overline{\mathbf{c}}, \overline{\mathbf{u}})$, then strong uniqueness is recovered around this specific state.

The next step by Bondesan and Briant has been a Cauchy theory for the Boltzmann system (5) having the property of being uniform with respect to the Knudsen number $\varepsilon$. Their result allows to prove that, in the limit $\varepsilon \rightarrow 0$, the Maxwell-Stefan 
equation are rigorously derived. The authors work in the three-dimensional torus $\mathbb{T}^{3}$.

The assumptions on the cross sections $B_{i j}$ at the kinetic level are the following:

(H1) All the $B_{i j}$ satisfy a symmetry property with respect to the interchange of the indices $i$ and $j$, i.e.:

$B_{i j}\left(\left|v-v_{*}\right|, \cos \theta\right)=B_{j i}\left(\left|v-v_{*}\right|, \cos \theta\right), \quad$ for all $v, v_{*} \in \mathbb{R}^{3}$ and for all $\theta \in \mathbb{R}$.

(H2) All cross sections $B_{i j}$ can be decomposed as the product of a kinetic part $\Phi_{i j} \geq 0$ and of an angular part $b_{i j} \geq 0$ :

$B_{i j}\left(\left|v-v_{*}\right|, \cos \theta\right)=\Phi_{i j}\left(\left|v-v_{*}\right|\right) b_{i j}(\cos \theta)$, for all $v, v_{*} \in \mathbb{R}^{3}$ and for all $\theta \in \mathbb{R}$.

(H3) The kinetic part is derived from an hard or a Maxwellian $(\gamma=0)$ potential:

$$
\Phi_{i j}\left(\left|v-v_{*}\right|\right)=C_{i j}^{\Phi}\left|v-v_{*}\right|^{\gamma}, \quad C_{i j}^{\Phi}>0, \quad \gamma \in[0,1], \quad \text { for all } v, v_{*} \in \mathbb{R}^{3} .
$$

(H4) The angular part satisfies Grad's angular cutoff in strong form [19]: there exists a constant $C>0$ such that

$$
0<b_{i j}(\cos \theta) \leq C|\sin \theta||\cos \theta|, \quad b_{i j}^{\prime}(\cos \theta) \leq C, \quad \theta \in[0, \pi] .
$$

Moreover,

$$
\inf _{\sigma_{1}, \sigma_{2} \in \mathbb{S}^{2}} \int_{\mathbb{S}^{2}} \min \left\{b_{i i}\left(\sigma_{1} \cdot \sigma_{3}\right), b_{i i}\left(\sigma_{2} \cdot \sigma_{3}\right)\right\} \mathrm{d} \sigma_{3}>0 .
$$

The precise statement of their theorem is the following [8]:

Theorem 2. Let the collision kernels $B_{i j}$ satisfy assumptions (H1)-(H2)-(H3)(H4), and let $\boldsymbol{\mu}$ be the unique global equilibrium of the mixture. Consider the local Maxwellian vector $\mathbf{M}^{\varepsilon}=\left(M_{1}^{\varepsilon}, \ldots, M_{I}^{\varepsilon}\right)$, where

$$
M_{1}^{\varepsilon}=c_{i}(t, x)\left(\frac{m_{i}}{2 \pi}\right)^{3 / 2} \exp \left(-\frac{m_{i}}{2}\left|v-\varepsilon u_{i}(t, x)\right|^{2}\right), \quad i=1, \ldots, \mathscr{I} .
$$

There exist $s_{0} \in \mathbb{N}^{*}, \bar{\delta}_{M S}>0$ and $\varepsilon_{0} \in(0,1]$ such that the following statements hold for any integer $s \geq s_{0}$.

i) There exist three sets of positive constants $\left(a_{\alpha}^{(s)}\right)_{\alpha}^{s},\left(b_{\alpha, k}^{(s)}\right)_{\alpha, k}^{s}$ and $\left(d_{\alpha, \beta}^{(s)}\right)_{\alpha, \beta}^{s}$ such that, for all $\varepsilon \in\left(0, \varepsilon_{0}\right]$, the following norms are equivalent:

$$
\|\cdot\|_{\mathscr{H} \mathscr{\varepsilon}_{\varepsilon}^{s}} \sim\left(\|\cdot\|_{L_{x, v}^{2}\left(\boldsymbol{\mu}^{-\frac{1}{2}}\right)}^{2}+\sum_{|\alpha| \leq s}\left\|\partial_{x}^{\alpha} \cdot\right\|_{L_{x, v}^{2}\left(\boldsymbol{\mu}^{-\frac{1}{2}}\right)}^{2}+\varepsilon_{\substack{|\alpha|+|\beta| \leq s \\|\beta| \geq 1}}\left\|\partial_{v}^{\beta} \partial_{x}^{\alpha} \cdot\right\|_{L_{x, v}^{2}\left(\boldsymbol{\mu}^{-\frac{1}{2}}\right)}^{2}\right)^{\frac{1}{2}} .
$$


ii) Let $\boldsymbol{L}$ be the vector of linearized mono-species Boltzmann operators around the global Maxwellian equilibrium of the mixture $\boldsymbol{\mu}$,

$$
\mathbf{T}^{\varepsilon}=\varepsilon^{-2} \boldsymbol{L}-\varepsilon^{-1} v \cdot \nabla_{x}
$$

and $\pi_{\mathbf{T}^{\varepsilon}}$ be the orthogonal projection onto $\operatorname{ker} \mathbf{T}^{\varepsilon}$ in $L^{2}\left(\mathbb{R}^{3}, \boldsymbol{\mu}^{-\frac{1}{2}}\right)$.

There exists $\delta_{B}>0$ such that, for all $\varepsilon \in\left(0, \varepsilon_{0}\right]$, for all $\delta_{M S} \in\left[0, \bar{\delta}_{M S}\right]$ and for any initial datum $\mathbf{f}^{\text {in }}$ in $H^{s}\left(\mathbb{T}^{3} \times \mathbb{R}^{3}, \boldsymbol{\mu}^{-\frac{1}{2}}\right)$ with

$$
\left\|\mathbf{f}^{\text {in }}\right\|_{\mathscr{H}_{\varepsilon}^{s}} \leq \delta_{B}, \quad\left\|\pi_{\mathbf{T}^{\varepsilon}}\left(\mathbf{f}^{\text {in }}\right)\right\|_{L_{x, v}^{2}\left(\boldsymbol{\mu}^{-\frac{1}{2}}\right)} \leq C \delta_{M S},
$$

for some positive constant $C>0$ independent of the parameters $\varepsilon$ and $\delta_{M S}$, there exists a unique $\mathbf{f} \in C^{0}\left(\mathbb{R}^{+} ; H^{s}\left(\mathbb{T}^{3} \times \mathbb{R}^{3}, \boldsymbol{\mu}^{-\frac{1}{2}}\right)\right)$ such that $\mathbf{F}^{\varepsilon}=\mathbf{M}^{\varepsilon}+\varepsilon \mathbf{f}$ is the unique weak solution of the Boltzmann multi-species equation (25). Moreover, if $\mathbf{F}^{\varepsilon, \text { in }}=\mathbf{M}^{\varepsilon, \text { in }}+\varepsilon \mathbf{f}^{\text {in }} \geq 0$, then $\mathbf{F}^{\varepsilon}(t, x, v) \geq 0$ almost everywhere on $\mathbb{R}^{+} \times \mathbb{T}^{3} \times$ $\mathbb{R}^{3}$. Finally, for any time $t \geq 0, \mathbf{F}^{\varepsilon}$ satisfies the stability property

$$
\left\|\mathbf{F}^{\varepsilon}-\mathbf{M}^{\varepsilon}\right\|_{\mathscr{H}_{\varepsilon}^{s}} \leq \varepsilon \delta_{B}, \quad \text { for all } \varepsilon \in\left(0, \varepsilon_{0}\right] .
$$

The constant $\delta_{B}$ is explicit and only depend on the number of species $\mathscr{I}$, on the

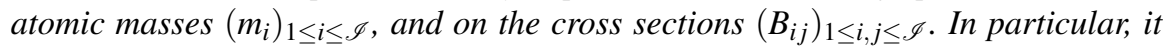
is independent of the parameters $\varepsilon$ and $\delta_{M S}$.

The strategy of proof consists in constructing perturbations of local Maxwellian states whose fluid quantities are perturbative solutions of the Maxwell-Stefan equations (29)-(31). Then, they plug the perturbation into the rescaled Boltzmann system (25). They obtain a system composed of four terms: the rescaled free transport operator, a linearized operator, a bilinear operator and a source term, which encodes the distance between the Maxwell-Stefan system and the fluid part of the perturbed Boltzmann equation.

However, the central spectral gap property cannot be directly recovered, because local Maxwellians are not local equilibria of the system, unless they share the same temperature and velocity (see Section 3). Nonetheless, the authors notice that the loss of the spectral gap has a lower order of magnitude. By introducing a modified Sobolev norm, they recover a coercivity property and close the energy estimates on the nonlinear terms.

Uniqueness is proved in a perturbative regime only.

A possible research direction consists in extending the previous strategy to the rigorous derivation of the Maxwell-Stefan equations in the non-isothermal setting. 


\section{The Maxwell-Stefan diffusion limit for reactive kinetic systems}

When chemical reactions are allowed, temperature may vary in space and time. Moreover, the structure of the target system depends on the relative order of magnitude of the reactive collisional terms with respect to the non-reactive ones.

In what follows, we will describe the situation of a quaternary reacting mixture, which is the most common situation considered in the literature. The extension to other types of mixture is straightforward.

Starting from the SRS kinetic model [24] for a mixture of four ideal gases undergoing a reversible chemical reaction of bimolecular type, Anwasia, Gonçalves and Soares derive in [4] the corresponding Maxwell-Stefan asymptotics in the diffusive scaling, in the vanishing limit of the mean free path, when all the collisional integrals have the same order of magnitude. The authors first define the reactive cross sections for the direct and reverse chemical reactions in terms of their threshold relative velocities. Let $d_{i}>0$ be the diameter of the particles of the $i$-th species $(i=1, \ldots, 4)$ and define, for any pair of species

$$
\sigma_{i j}^{2}=\frac{1}{4}\left(d_{i}+d_{s}\right)^{2}, \quad i=1, \ldots, 4, \quad j=1, \ldots, 4 .
$$

Then

$$
\sigma_{12}^{\prime 2}=\left\{\begin{array}{ll}
\beta_{12} \sigma_{12}^{2} & \left\langle\varepsilon, v_{1}-v_{2}\right\rangle \geq \Xi_{12} \\
0 & \left\langle\varepsilon, v_{1}-v_{2}\right\rangle<\Xi_{12}
\end{array} \quad \sigma_{34}^{\prime 2}= \begin{cases}\beta_{34} \sigma_{34}^{2} & \left\langle\varepsilon, v_{3}-v_{4}\right\rangle \geq \Xi_{34} \\
0 & \left\langle\varepsilon, v_{3}-v_{4}\right\rangle<\Xi_{34}\end{cases}\right.
$$

where the coefficients $\beta_{i j} \in[0,1]$ represent the fraction of colliding pairs with enough kinetic energy to produce a chemical reaction.

By neglecting external forces, the SRS kinetic equations are given by

$$
\frac{\partial f_{i}}{\partial t}+v_{i} \cdot \nabla_{x} f_{i}=J_{i}, \quad \text { in } \quad \mathbb{R}_{+} \times \Omega \times \mathbb{R}^{3}
$$

with $J_{i}=J_{i}^{E}+J_{i}^{R}$, for $i=1,2,3,4$. For each species, $J_{i}^{E}$ denotes the elastic collision operator and $J_{i}^{R}$ the reactive collision operator. Their precise form is

$$
\begin{gathered}
J_{i}^{E}=\sigma_{i i}^{2} \int_{\mathbb{R}^{3}} \int_{\mathbb{S}_{+}^{2}}\left[f_{i}^{\prime} f_{i_{*}}^{\prime}-f_{i} f_{i_{*}}\right]\left\langle\varepsilon, v_{i}-v_{i_{*}}\right\rangle d \varepsilon d v_{i_{*}} \\
+\sum_{\substack{s=1 \\
s \neq i}}^{4} \sigma_{i s}^{2} \int_{\mathbb{R}^{3}} \int_{\mathbb{S}_{+}^{2}}\left[f_{i}^{\prime} f_{s}^{\prime}-f_{i} f_{s}\right]\left\langle\varepsilon, v_{i}-v_{s}\right\rangle d \varepsilon d v_{s} \\
\quad-\beta_{i j} \sigma_{i j}^{2} \int_{\mathbb{R}^{3}} \int_{\mathbb{S}_{+}^{2}}\left[f_{i}^{\prime} f_{j}^{\prime}-f_{i} f_{j}\right] \Theta\left(\left\langle\varepsilon, v_{i}-v_{j}\right\rangle-\Xi_{i j}\right)\left\langle\varepsilon, v_{i}-v_{j}\right\rangle d \varepsilon d v_{j}, \\
J_{i}^{R}=\beta_{i j} \sigma_{i j}^{2} \int_{\mathbb{R}^{3}} \int_{\mathbb{S}_{+}^{2}}\left[\left(\frac{\mu_{i j}}{\mu_{k l}}\right)^{2} f_{k}^{\circ} f_{l}^{\circ}-f_{i} f_{j}\right] \Theta\left(\left\langle\varepsilon, v_{i}-v_{j}\right\rangle-\Xi_{i j}\right)\left\langle\varepsilon, v_{i}-v_{j}\right\rangle d \varepsilon d v_{j},
\end{gathered}
$$


where $f_{i}^{\prime}=f\left(t, x, v_{i}^{\prime}\right), f_{i *}^{\prime}=f\left(t, x, v_{i *}^{\prime}\right), f_{s}^{\prime}=f\left(t, x, v_{s}^{\prime}\right), f_{k}^{\circ}=f\left(t, x, v_{k}^{\circ}\right), f_{l}^{\circ}=f\left(t, x, v_{l}^{\circ}\right)$, and $\Theta$ is the Heaviside step function.

Then Anwasia, Gonçalves and Soares prove that, in the limit, the concentrations $c_{i}$ and the diffusive fluxes $\mathbf{J}_{i}$ satisfy an equation of Maxwell-Stefan type:

$$
\left\{\begin{array}{c}
\frac{\partial c_{i}}{\partial t}+\nabla_{x} \cdot \mathbf{J}_{\mathbf{i}}=\sigma_{i j}^{2}\left(\frac{2 \pi \mu_{i j}}{k_{B} T}\right)^{\frac{1}{2}}\left[\left(\frac{\mu_{i j}}{\mu_{k l}}\right)^{\frac{1}{2}} c_{k} c_{l} \exp \left(\frac{E}{k_{B} T}\right)-c_{i} c_{j}\right] \\
\times\left[\frac{2 k_{B} T}{\mu_{i j}} \Gamma\left(2, z_{i}^{*}\right)-\left(\frac{\Xi_{i j}}{c_{0}}\right)^{2} \Gamma\left(1, z_{i}^{*}\right)\right] \\
\nabla_{x} c_{i}=\frac{32}{3} \sum_{\substack{s=1 \\
s \neq i}}^{4} \sigma_{i s}^{2}\left(\frac{2 \pi \mu_{i j}}{k_{B} T}\right)^{\frac{1}{2}}\left(c_{i} \mathbf{J}_{s}-c_{s} \mathbf{J}_{i}\right),
\end{array}\right.
$$

with

$$
\mu_{i j}=\frac{m_{i} m_{j}}{m_{i}+m_{j}}, \quad z_{i}^{*}=\frac{\mu_{i j}}{2 k_{B} T}\left(\frac{\Xi_{i j}}{c_{0}}\right)^{2},
$$

where $E$ is the reaction heat defined by the balance of chemical binding energies (18), $\Gamma$ denotes the incomplete gamma function, $c_{0}$ denotes the characteristic speed of sound in the mixture at a reference temperature $T_{0}, \zeta_{i}$ is the activation energy for each of the species and $\Xi_{i j}=\sqrt{2 \zeta_{i} / \mu_{i j}}$ is the threshold relative velocity.

It is worth noticing that no influence of the chemical reaction appears in the limiting Maxwell-Stefan equations written above.

In [3], the same authors modify the scaling, by supposing that both mechanical collisions and chemical reactions have comparable relaxation times, much smaller than the characteristic time of the flow. The reactive Maxwell-Stefan equations under this scaling have the form:

$$
\nabla_{x}\left(c_{i} T\right)=-\sum_{\substack{s=1 \\ s \neq i}}^{4}\left(\frac{c_{s} \mathbf{J}_{s}-c_{i} \mathbf{J}_{s}}{D_{i s}}\right)+\frac{c_{j} \mathbf{J}_{i}-c_{i} \mathbf{J}_{j}}{D_{i j}}+\frac{c_{l} \mathbf{J}_{k}}{D_{k}}+\frac{c_{k} \mathbf{J}_{l}}{D_{l}}-\frac{c_{j} \mathbf{J}_{i}}{D_{i}}-\frac{c_{i} \mathbf{J}_{j}}{D_{j}},
$$

where the indices $(i, j, k, l)$ take the values $(1,2,3,4),(2,1,4,3),(3,4,1,2)$ or $(4,3,2,1)$, and the quantities $D_{i s}, D_{i j}, D_{k}, D_{l}, D_{i}$ are explicitly computed diffusion coefficients, based on the kinetic parameters of the model.

We conclude this section with the formal derivation of the Maxwell-Stefan asymptotics starting from the kinetic model for reactive gases by Desvillettes, Monaco and Salvarani [16] whose precise form is described in Section 4, under the standard diffusive scaling and when mechanical collisions are predominant with respect to reacting collisions.

The formal limit as $\varepsilon \rightarrow 0$, when the angular collision kernels of both the elastic bi-species and the reactive operators are odd functions of $\cos \theta$, has been studied by Anwasia, Bisi, Salvarani and Soares [2]. The resulting target equations have the structure of a system of balance equations coupled with the corresponding 
temperature-dependent flux-gradient relationships and with an equation describing the energy balance:

$$
\begin{gathered}
\frac{\partial c_{i}}{\partial t}+\nabla_{x} \cdot J_{i}=-\lambda_{i} A \quad i=1,2,3,4 \\
\nabla_{x}\left(c_{i} k_{B} T\right)=-\sum_{\substack{j=1 \\
j \neq i}}^{4} \frac{c_{j} \mathbf{J}_{i}-c_{i} \mathbf{J}_{j}}{D_{i j}} \quad i=1,2,3,4 \\
\frac{\partial}{\partial t}\left[\sum_{i=1}^{4} c_{i}\left(\frac{3}{2} k_{B} T+\frac{q_{i}^{*}(T)}{q_{i}(T)}\right)\right]+\nabla_{x} \cdot\left[\sum_{i=1}^{4}\left(\frac{5}{2} k_{B} T+\frac{q_{i}^{*}(T)}{q_{i}(T)}\right) J_{i}\right]=-E A,
\end{gathered}
$$

where the production term $A$ is given by

$$
\begin{aligned}
A & =\left[\left(\frac{m_{3} m_{4}}{m_{1} m_{2}}\right)^{3 / 2} \frac{c_{1} c_{2}}{q_{1}(T(t, x)) q_{2}(T(t, x))} \exp \left(-\frac{E}{k_{B} T(t, x)}\right)\right. \\
& \left.-\frac{c_{3} c_{4}}{q_{3}(T(t, x)) q_{4}(T(t, x))}\right] \frac{4}{\sqrt{\pi}\left(m_{3} m_{4}\right)^{2}} \Gamma\left(\frac{\gamma+2}{2}\right)\left(\frac{2 k_{B} T}{\mu_{34}}\right)^{(\gamma-1) / 2} \\
& \times \int_{0}^{+\infty} \int_{0}^{+\infty} \int_{0}^{1} \int_{0}^{1} \int_{\mathbb{S}^{2}} \exp \left(-\frac{I+I_{4}}{k_{B} T}\right) \Phi^{\text {react }}\left(I, I_{4}, R, r\right) \\
& \times b^{\text {react }}(\cos \theta) \cos \theta(1-R) \mathrm{d} \sigma \mathrm{d} r \mathrm{~d} R \mathrm{~d} I_{4} \mathrm{~d} I,
\end{aligned}
$$

and the diffusion coefficients $D_{i j}$ are

$$
\begin{aligned}
& \frac{1}{D_{i j}}=\frac{4}{3 \sqrt{\pi}} \mu_{i j}^{(3-\gamma) / 2}\left(2 k_{B} T(t, x)\right)^{(\gamma-1) / 2} \frac{1}{q_{i}(T(t, x)) q_{j}(T(t, x))} \\
& \times \Gamma\left(\frac{\gamma+4}{2}\right) \int_{0}^{+\infty} \int_{0}^{+\infty} \int_{0}^{1} \int_{0}^{1} \int_{\mathbb{S}^{2}} \exp \left(-\frac{I+I_{j}}{k_{B} T(t, x)}\right) \Phi_{i j}\left(I, I_{j}, R, r\right) \\
& \quad \times \cos \theta(1-R) b_{i j}(\cos \theta) \mathrm{d} \sigma \mathrm{d} r \mathrm{~d} R \mathrm{~d} I_{j} \mathrm{~d} I .
\end{aligned}
$$

In the previous formulas, $\Gamma$ denotes the complete Gamma function and the quantities $q_{i}$ are given by (24), whereas

$$
q_{i}^{*}(T(t, x))=\int_{0}^{+\infty} I \varphi_{i}(I) \exp \left(-\frac{I}{k_{B} T(t, x)}\right) \mathrm{d} I .
$$

The structure of these equations takes into account the effects of the chemical reactions and their consequences with respect to the evolution of the temperature of the mixtures. We underline that they are compatible with the energy law of polyatomic gases and the law of mass action. Indeed, at the equilibrium, it is possible to deduce the law of mass action 


$$
\frac{c_{1} c_{2}}{c_{3} c_{4}}=\left(\frac{m_{1} m_{2}}{m_{3} m_{4}}\right)^{3 / 2} \frac{q_{1}(T(t, x)) q_{2}(T(t, x))}{q_{3}(T(t, x)) q_{4}(T(t, x))} \exp \left(\frac{E}{k_{B} T(t, x)}\right),
$$

which depends on the choice of the functional forms of the weights $\varphi_{i}$. The choice of these weights can be adapted to the physical situation of the mixture.

\section{Some related research directions}

Some other articles consider problems with a strong relationship with the subject of this note.

Bondesan, Boudin and Grec [6] derive a numerical method for the Euler equations describing a mixture, stable with respect to the relaxation parameter, whose limit is the Maxwell-Stefan system.

Briant and Grec have studied in [15] the asymptotics of the Boltzmann system (5)-(6) in a framework different from the one studied in [8]. They suppose that, at the leading order, the species velocities are identical and obtain a cross-diffusion system of Fick type, not equivalent to the Maxwell-Stefan systems because the two matrices linking fluxes and concentrations are not invertible.

A relationship between the Fick diffusion and Maxwell-Stefan diffusion has been investigated by Salvarani and Soares in [27]. They rigorously prove the relaxation of the Maxwell-Stefan system, together with the equimolar closure relationship (2), towards a system of uncoupled linear diffusion equations of Fickian type.

Acknowledgements Work supported by the ANR projects Kimega (ANR-14-ACHN-0030-01) and by the Italian Ministry of Education, University and Research (Dipartimenti di Eccellenza program 2018-2022, Dipartimento di Matematica 'F. Casorati', Università degli Studi di Pavia). The author thanks the organizers of the series of conferences PSPDE for their generous hospitality and support. He moreover thanks the anonymous referees for their useful comments.

\section{References}

1. B. Anwasia. The Maxwell-Stefan diffusion limit of a hard-sphere kinetic model for mixtures. arXiv preprint arXiv:2004.11079.

2. B. Anwasia, M. Bisi, F. Salvarani, and A. J. Soares. On the Maxwell-Stefan diffusion limit for a reactive mixture of polyatomic gases in non-isothermal setting. Kinetic \& Related Models, $13: 63,2020$

3. B. Anwasia, P. Gonçalves, and A. J. Soares. On the formal derivation of the reactive MaxwellStefan equations from the kinetic theory. EPL (Europhysics Letters), 129(4):40005, 2020.

4. B. Anwasia, P. Gonçalves, and A. J. Soares. From the simple reacting sphere kinetic model to the reaction-diffusion system of Maxwell-Stefan type. Commun. Math. Sci., 17(2):507-538, 2019.

5. L. Boltzmann. Weitere Studien über das Wärmegleichgewicht unter Gasmoläkulen. Sitzungsberichte der Akademie der Wissenschaften, 66:275-370, 1872. 
6. A. Bondesan, L. Boudin, and B. Grec. A numerical scheme for a kinetic model for mixtures in the diffusive limit using the moment method. Numer. Methods Partial Differential Equations, 35(3):1184-1205, 2019.

7. A. Bondesan and M. Briant. Perturbative Cauchy theory for a flux-incompressible MaxwellStefan system in a non-equimolar regime. arXiv preprint arXiv:1910.03279.

8. A. Bondesan and M. Briant. Stability of the Maxwell-Stefan system in the diffusion asymptotics of the Boltzmann multi-species equation. arXiv preprint arXiv:1910.08357.

9. C. Borgnakke and P. S. Larsen. Statistical collision model for Monte Carlo simulation of polyatomic gas mixture. Journal of Computational Physics, 18(4):405 - 420, 1975.

10. D. Bothe. On the Maxwell-Stefan approach to multicomponent diffusion. In Parabolic problems, volume 80 of Progr. Nonlinear Differential Equations Appl., pages 81-93. Birkhäuser/Springer Basel AG, Basel, 2011.

11. L. Boudin, B. Grec, and V. Pavan. The Maxwell-Stefan diffusion limit for a kinetic model of mixtures with general cross sections. Nonlinear Anal., 159:40-61, 2017.

12. L. Boudin, B. Grec, and F. Salvarani. A mathematical and numerical analysis of the MaxwellStefan diffusion equations. Discrete Contin. Dyn. Syst. Ser. B, 17(5):1427-1440, 2012.

13. L. Boudin, B. Grec, and F. Salvarani. The Maxwell-Stefan diffusion limit for a kinetic model of mixtures. Acta Appl. Math., 136:79-90, 2015.

14. J.-F. Bourgat, L. Desvillettes, P. Le Tallec, and B. Perthame. Microreversible collisions for polyatomic gases and Boltzmann's theorem. European J. Mech. B Fluids, 13(2):237-254, 1994.

15. M. Briant and B. Grec. Rigorous derivation of the Fick cross-diffusion system from the multispecies Boltzmann equation in the diffusive scaling. arXiv preprint arXiv:2003.07891.

16. L. Desvillettes, R. Monaco, and F. Salvarani. A kinetic model allowing to obtain the energy law of polytropic gases in the presence of chemical reactions. Eur. J. Mech. B Fluids, 24(2):219-236, 2005.

17. A. Ern and V. Giovangigli. Multicomponent transport algorithms, volume 24 of Lecture Notes in Physics. New Series M: Monographs. Springer-Verlag, Berlin, 1994.

18. V. Giovangigli. Multicomponent flow modeling. Modeling and Simulation in Science, Engineering and Technology. Birkhäuser Boston Inc., Boston, MA, 1999.

19. H. Grad. Principles of the kinetic theory of gases. In Handbuch der Physik (herausgegeben von S. Flügge), Bd. 12, Thermodynamik der Gase, pages 205-294. Springer-Verlag, BerlinGöttingen-Heidelberg, 1958.

20. H. Grad. Asymptotic theory of the Boltzmann equation. II. In Rarefied Gas Dynamics (Proc. 3rd Internat. Sympos., Palais de l'UNESCO, Paris, 1962), Vol. I, pages 26-59. Academic Press, New York, 1963.

21. M. Groppi and G. Spiga. Kinetic approach to chemical reactions and inelastic transitions in a rarefied gas. Journal of Mathematical chemistry, 26(1-3):197-219, 1999.

22. H. Hutridurga and F. Salvarani. Maxwell-Stefan diffusion asymptotics for gas mixtures in non-isothermal setting. Nonlinear Anal., 159:285-297, 2017.

23. H. Hutridurga and F. Salvarani. On the Maxwell-Stefan diffusion limit for a mixture of monatomic gases. Math. Methods Appl. Sci., 40(3):803-813, 2017.

24. M. T. Marron. Simple collision theory of reactive hard spheres. J. Chem. Phys, 52(8):40604061, 1970.

25. J. C. Maxwell. On the dynamical theory of gases. Phil. Trans. R. Soc., 157:49-88, 1866.

26. T. F. Morse. Kinetic model equations for a gas mixture. Phys. Fluids, 7:908-918, 1964.

27. F. Salvarani and A. J. Soares. On the relaxation of the Maxwell-Stefan system to linear diffusion. Appl. Math. Lett., 85:15-21, 2018.

28. L. Sirovich. Kinetic modeling of gas mixtures. Phys. Fluids, 5(8):908-918, 1962.

29. J. Stefan. Über das Gleichgewicht und die Bewegung insbesondere die Diffusion von Gasgemengen. Akad. Wiss. Wien, 63:63-124, 1871.

30. R. Taylor and R. Krishna. Multicomponent mass transfer, volume 2. John Wiley \& Sons, 1993.

31. C. Villani. A review of mathematical topics in collisional kinetic theory. In Handbook of mathematical fluid dynamics, Vol. I, pages 71-305. North-Holland, Amsterdam, 2002. 